\title{
Influence of temperature and viscosity on anthracene rotational diffusion in organic solvents: Molecular dynamics simulations and fluorescence anisotropy study
}

\author{
Gouri S. Jas, ${ }^{\text {a) }}$ Yan Wang, Steven W. Pauls, Carey K. Johnson, and Krzysztof Kuczera ${ }^{\text {b) }}$ \\ Departments of Chemistry and Biochemistry, University of Kansas, Lawrence, Kansas 66045
}

(Received 25 April 1997; accepted 29 August 1997)

\begin{abstract}
Molecular dynamics simulations and fluorescence anisotropy decay measurements are used to investigate the rotational diffusion of anthracene in two organic solvents-cyclohexane and 2-propanol-at several temperatures. Molecular dynamics simulations of $1 \mathrm{~ns}$ length were performed for anthracene in cyclohexane (at 280, 296, and $310 \mathrm{~K}$ ) and in 2-propanol (at $296 \mathrm{~K}$ ). The calculated time constants for reorientation of the short in-plane axis were 7-9 and 11-16 ps at 296 $\mathrm{K}$ in cyclohexane and 2-propanol, respectively, in excellent agreement with corresponding fluorescence depolarization measurements of 8 and 14 ps. The measured rotational reorientation times and the calculated average rotational diffusion coefficients varied in accord with DebyeStokes-Einstein theory. Their magnitudes were close to values predicted for an ellipsoid of shape and size equivalent to an anthracene molecule, and exhibited predictable variation with external conditions-increasing with temperature and decreasing with solvent viscosity. However, analysis of the calculated rotational diffusion coefficients for the individual molecular axes gave a more complex picture. The diffusion was highly anisotropic and changes in temperature and solvent type led to nonuniform variation of the diffusion coefficients. The nature of these changes was rationalized based on analysis of variation of solvation patterns with temperature and solvent. (C) 1997 American Institute of Physics. [S0021-9606(97)51945-3]
\end{abstract}

\section{INTRODUCTION}

Translational and rotational diffusion studies provide valuable information on molecular motions in liquids, probing mobility, interactions, structural transitions and chemical reactions. ${ }^{1-6}$ The rotational relaxation of fluorescent and spin probes is also an important tool in studying structure and dynamics of macromolecular systems, such as proteins or membranes. ${ }^{7-12}$ For these reasons molecular diffusion has attracted significant scientific interest, and has been the object of numerous theoretical, experimental and simulation studies.

Investigations of molecular diffusion follow several paths. One path involves use of hydrodynamic theory and the relationship between diffusion and friction. ${ }^{13-17}$ Rotational diffusion coefficients for molecules modeled as regular geometric shapes moving through a continuous viscous medium were given analytically for spheres ${ }^{18}$ and triaxial ellipsoids under stick conditions. ${ }^{19,20}$ Numerical results were obtained for biaxial $^{21,22}$ and triaxial ellipsoids under slip conditions. ${ }^{23,24}$ Translational diffusion coefficients were determined analytically for a sphere ${ }^{18}$ and a triaxial ellipsoid ${ }^{19,20}$ under stick conditions. More recently numerical results for arbitrary shapes have also been obtained. ${ }^{25-29} \mathrm{An}-$ other path involves extracting diffusion coefficients from experimental observables, such as fluorescence depolarization, ${ }^{30,31}$ light scattering, ${ }^{32,33}$ forced Rayleigh scattering, ${ }^{34}$ electric birefringence ${ }^{35}$ and nuclear magnetic

\footnotetext{
${ }^{\text {a) }}$ Current address: NIH, NIDDK, Bldg. 5, 9000 Rockville Pike, Bethesda, Maryland 20892.

b) Author to whom correspondence should be addressed; electronic mail: kuczera@tedybr.chem.ukans.edu
}

resonance (NMR) spectral parameters. ${ }^{5,36-38}$ Finally, the advent of computer simulations has opened the door to direct simulations of diffusion, which enable both validation of theoretical models and insight into atomic-level phenomena responsible for experimentally observed effects.

Numerous simulations of translational and rotational diffusion in molecular systems have been performed, on systems ranging from pure liquids ${ }^{39-41}$ to proteins. ${ }^{42}$ The majority of the previous simulations focus on pure liquid systems, advantageous for their relative simplicity and enhanced sampling. Only a small number of studies, using relatively short simulations, have been performed for solutions. ${ }^{42-44}$

In this article we present a systematic study of the rotational diffusion of anthracene in organic solvents. In the computational part of the study we calculate the rotational diffusion coefficients from $1 \mathrm{~ns}$ length molecular dynamics simulations of anthracene in cyclohexane (at 280, 296, and $310 \mathrm{~K}$ ) and isopropanol (at $296 \mathrm{~K}$ ). Cyclohexane and isopropanol have been chosen as examples of an alkane and an alcohol solvent, differing not only in room temperature viscosity but also in shape, size and self-interactions. Our study is designed to uncover the role of these different properties on the details of anthracene rotational diffusion. We use several different calculation methods to obtain both the average diffusion constants and diffusion rates around each of the molecular axes. In each simulation we also determine the average three-dimensional solvent distribution around the anthracene molecule. In the spectroscopic part of the paper we present fluorescence anisotropy decay measurements for anthracene in the same solvents as were employed in the simulations. Finally, we compare the detailed simulation re- 
sults with hydrodynamic theory predictions and with fluorescence anisotropy decay measurements.

The simulated decay times for reorientation of the transition dipole axis are in good agreement with experimental data in previous ${ }^{33,45,56}$ and in the present work. Also, the calculated variation of average diffusion coefficients with solvent viscosity and temperature is in accord with simple hydrodynamic theory. Additionally, our simulations indicate that changes in temperature and solvent type lead to nonuniform variations of the diffusion coefficients along the individual molecular axes. The nature of these changes is rationalized based on analysis of variations of microscopic solvation patterns.

Anthracene is a chromophoric model compound which has been extensively studied. Numerous spectroscopic and theoretical investigations of the ground and excited state have been carried out in order to understand the photophysics of anthracene. ${ }^{46-55}$ The rotational motion of anthracene in organic solvents has previously been studied with IR/UV double resonance, ${ }^{56}$ depolarized light scattering and fluorescence anisotropy decay. ${ }^{33}$

Our study, by providing new microscopic details, theoretical analysis and experimental data, is aimed at improving the basic understanding of molecular diffusion of anthracene in particular and also of related probe molecules. Our results suggest that the simple behavior of the average rotational correlation time, often observed for probe molecules such as anthracene ${ }^{57}$ may disguise interesting and informative variations in the rotational diffusion coefficients for specific molecular axes. These variations may reflect specific solutesolvent interactions and the microscopic effects of solvent structure. Our results should be a helpful reference for analyzing experimental and simulation results of tethered chromophoric probes. Our study of the relatively rigid anthracene system is also a good starting point on the way to simulating diffusion of more complex, flexible systems of biological interest, such as peptides.

\section{METHODS}

\section{A. Fluorescence anisotropy decay measurements}

In the fluorescence depolarization experiments reported here, pulses at $730 \mathrm{~nm}$ were generated by a cavity-dumped rhodamine 700 dye laser, which was synchronously pumped by the second harmonic from a Nd:YAG laser (Coherent Antares). Cavity-dumped pulses at a repetition rate of 3.975 $\mathrm{MHz}$ were frequency-doubled in a BBO crystal to provide an excitation wavelength of $365 \mathrm{~nm}$. The excitation beam was focused near the front face of a $1.0 \mathrm{~cm} \times 1.0 \mathrm{~cm}$ quartz sample cell by a $10-\mathrm{cm}$ lens. Emission photons collected at $90^{\circ}$ were passed through a polarizer and a polarization scrambler, focused into a subtractive-dispersion double monochromator (American Holographic DB-10), and detected by a microchannel-plate photomultiplier tube (Hamamatsu R3809U). Fluorescence decay profiles were acquired by time-correlated single photon counting (Tennelek), with the time-to-amplitude converter (TAC) operated in reverse mode, i.e., the TAC start pulse was derived from the fluorescence photons and the stop pulse from the a laser reference pulse. The instrumental response was characterized by a full width at half-maximum (FWHM) of about 20 ps.

Zone-refined (99\%) anthracene was purchased from Aldrich and used as received. Spectral grade solvents 2propanol $(99.9 \%)$ and cyclohexane $(99 \%)$ were purchased from Aldrich and showed no evidence of significant fluorescence under our experimental conditions. These solvents were bubbled with nitrogen gas for 40 min prior to use. Concentrations of anthracene solutions were kept below 1 $\times 10^{-5} \mathrm{M}$ to reduce the possibility of self-quenching.

The initial anisotropy of anthracene with $365 \mathrm{~nm}$ excitation was found to be 0.198 in propylene glycol at $-15^{\circ} \mathrm{C}$. Polarized fluorescence decay curves were collected separately and tail-matched by collecting until the same signal was acquired over a time range in the tail of each decay where the fluorescence depolarization was essentially complete. The parallel and perpendicular curves were then deconvoluted simultaneously by nonlinear least squares with the Levenberg-Marquardt algorithm. ${ }^{58}$ All data were fit with one fluorescence lifetime $(\sim 4.7 \mathrm{~ns})$ and one rotational correlation time, with the initial anisotropy fixed at a value of 0.198 . Two or three fluorescence depolarization scans were collected for each solvent and temperature, yielding independent measurements of the correlation time. Uncertainties in the rotational correlation times, as estimated from the variance of the data with the Student $t$ correction at the $90 \%$ confidence limit, were \pm 6 ps or less. The rotational correlation times determined in this way also depend on the value of the initial anisotropy. An uncertainty of \pm 0.01 in the initial anisotropy value introduces an additional uncertainty of about $\pm 5 \mathrm{ps}$ in the rotational correlation times, resulting in a net uncertainty of \pm 8 ps or less. However, the rotational correlation time determined for each solvent and temperature is affected in the same direction by variations in the value at which the initial anisotropy is fixed. Consequently, the uncertainty in the initial anisotropy does not affect the relative variations in rotational correlation times with solvent and temperature.

\section{B. Force field parameterization}

The CHARMM program (Version 22) was used in the simulations ${ }^{59}$ with the version 22 all-hydrogen topology and parameters ${ }^{60}$ in which all atoms are treated explicitly. For anthracene all carbons were assigned to the existing CHARMM aromatic carbon type CA, and all hydrogens to the existing type HP. ${ }^{59}$ This assignment defined all force field parameters for internal strain and van der Waals potential energy terms. Atomic charges were obtained from the Mulliken population analysis of the HF/6-31G* optimized $D_{2 h}$ structure of anthracene ${ }^{55}$ scaled down by 0.9 to correct for systematic error. ${ }^{61,62}$ With these parameters, the CHARMM optimized geometry of anthracene is found to be in good agreement with the crystal structure, with bond length deviations below $0.02 \AA$ and bond angle deviations below $2^{\circ}$. This structure and our convention for labeling anthracene molecular axes are described in Fig. 1. 


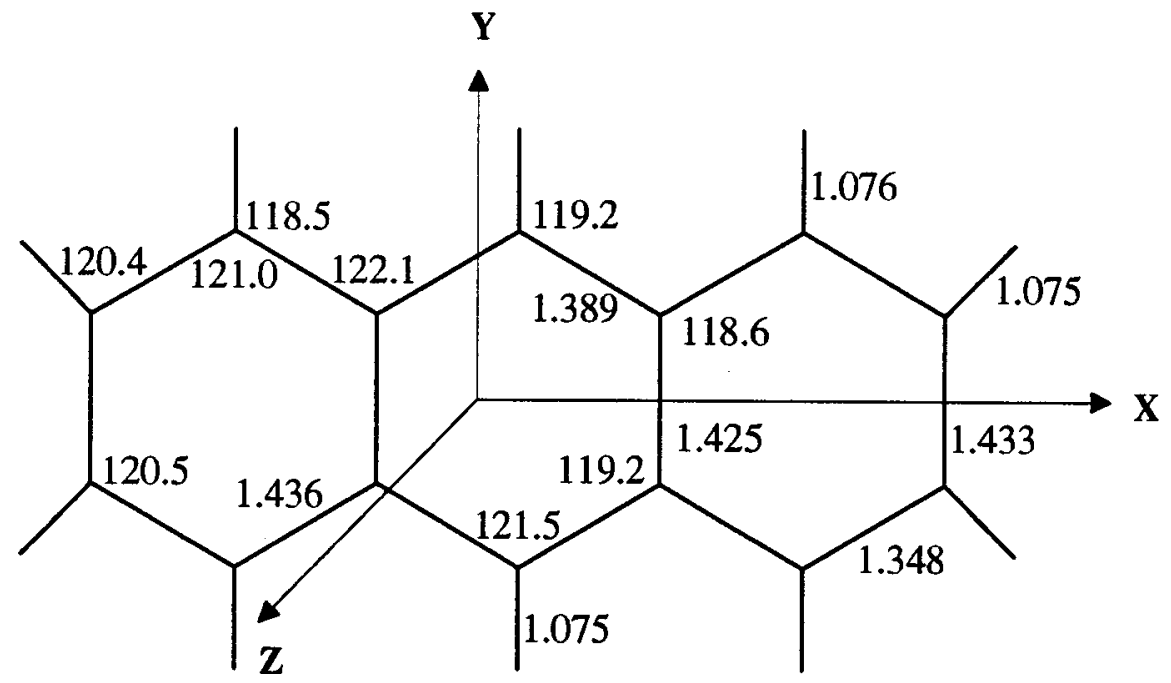

FIG. 1. Geometry of anthracene and molecular axis system. Bond lengths in angstrom, angles in degrees.

For cyclohexane the existing CHARMM aliphatic carbons (CT2) and hydrogens (HA) were used with the standard aliphatic charges -0.18 of on methylene carbons and 0.09 on hydrogens (all atomic charges are in atomic units). For 2propanol the CHARMM topology, parameters and atomic charges from the threonine sidechain were used; with atom types CT1 for the CH carbon, CT3 for the methyl group carbons, $\mathrm{OH} 1$ for the hydroxyl oxygen, HA for the aliphatic hydrogens and $\mathrm{H}$ for the hydroxyl hydrogen. ${ }^{59}$ The atomic charges were 0.14 on $\mathrm{CT} 1,-0.66$ on $\mathrm{OH} 1,0.43$ on $\mathrm{H}$, -0.27 on CT3, and 0.09 on HA. ${ }^{59}$ The properties of pure liquid cyclohexane and isopropanol modeled by these parameters are in good agreement with with experimental data. ${ }^{63}$

\section{Molecular dynamics simulations protocol}

Four $1 \mathrm{~ns}$ trajectories were generated, corresponding to anthracene in cyclohexane at 280, 296, and $310 \mathrm{~K}$, and anthracene in 2-propanol at $296 \mathrm{~K}$. The initial structure of anthracene was taken from the CHARMM optimized geometry. Truncated cubic cells with 108 cyclohexanes and 1532 propanols were equilibrated at $296 \mathrm{~K}$ and constant volume, with densities corresponding to $1 \mathrm{~atm}$ pressure (densities used: $0.7785 \mathrm{~g} / \mathrm{cm}^{3}$ for cyclohexane, ${ }^{64} 0.7855 \mathrm{~g} / \mathrm{cm}^{3}$ for 2-propanol ${ }^{64}$ ). After replacing one solvent molecule with anthracene and correcting for the difference in molecular volume between solute and solvent at $296 \mathrm{~K}$, the cyclohexane solution system thus contained one anthracene and $107 \mathrm{cy}-$ clohexane molecules in a truncated cubic cell based on a cube of side $33.8366 \AA$, while the isopropanol system contained one anthracene and 152 isopropanol molecules in a cell based on a cube of side $33.8966 \AA$. The CHARMM van der Waals volume of anthracene $\left(223 \AA^{3}\right)$ was used in the correction. An analogous procedure was performed for the cyclohexane simulations at 280 and $310 \mathrm{~K}$; using pure cyclohexane densities of 0.7905 and $0.7625 \mathrm{~g} / \mathrm{cm}^{3},{ }^{65}$ respectively led to simulation cells based on cubes of $33.5318 \AA$ at $280 \mathrm{~K}$ and $33.9387 \AA$ at $310 \mathrm{~K}$. In all molecular dynamics (MD) simulations SHAKE constraints ${ }^{66}$ were introduced on the bonds involving hydrogen atoms, allowing use of a $2 \mathrm{fs}$ time step. ${ }^{67}$ In energy calculations a $12.0 \AA$ atom based nonbonded cutoff distance was used, with a switching function between 10.0 and $12.0 \AA$ for van der Waals terms and a shift function at $12.0 \AA$ for electrostatics in order to eliminate discontinuities due to the cutoff. Each simulation system was prepared by energy minimization, 10 ps of heating by random velocity assignments at stepwise increasing temperatures and 10 ps equilibration at the target temperature (280, 296 , and $310 \mathrm{~K}$ in cyclohexane) and $296 \mathrm{~K}$ in 2-propanol, followed by $1 \mathrm{~ns}$ trajectory generation. The Verlet algorithm was used for integration of equations of motion in all simulations. ${ }^{68}$ Periodic boundary conditions were applied and the simulation conditions corresponded to the microcanonical ensemble. The average temperature fluctuations were $4 \mathrm{~K}$ in all four $1 \mathrm{~ns}$ trajectories. Coordinates and velocities of all atoms were saved every $0.05 \mathrm{ps}$ for analysis.

\section{Rotational diffusion analysis}

Rotational diffusion coefficients of anthracene, $D_{r}$, were calculated using four methods, based on reorientation of the molecule as a whole, reorientation of the individual molecular axes and angular velocity correlation functions (AVACF) in the laboratory (LAB) and molecular (MOL) frames. In the first method the expression ${ }^{69} D_{r}=\left\langle\Delta \theta^{2}(t)\right\rangle / 6 t$ was used, where $\Delta \theta(t)$ is the angle of rotation of the molecule-fixed frame during time $t$ and $\langle\ldots\rangle$ is an average over initial positions. The reorientation angle between two anthracene structures was calculated by overlaying them in the root-meansquare sense using the CHARMM program. ${ }^{58}$ We calculated $D_{r}$ from the slope of $\left\langle\Delta \theta^{2}(t)\right\rangle$ in its region of linear behavior, 5-40 ps.

In the LAB AVACF method, the angular velocity time series $\vec{\omega}(t)$ of rotation around the anthracene CM is calculated from the trajectory as $\vec{\omega}(t)=\mathbf{I}(t)^{-1} \cdot \vec{L}(t)$, where $\mathbf{I}(t)^{-1}$ is the inverse of the instantaneous moment of inertia tensor and $\vec{L}(t)$ is the angular momentum relative to the 
molecular CM. The diffusion coefficient is then calculated from the integral as follows: ${ }^{70,71}$

$$
D_{r}=\frac{1}{3} \int_{0}^{\tau_{r}} d t\langle\vec{\omega}(0) \cdot \vec{\omega}(t)\rangle .
$$

The calculated $D_{r}$ values exhibit some dependence on the upper limit $\tau_{r}$ of the integral. To eliminate this dependence, the average value of the integral for $\tau_{r}$ between 5 and $15 \mathrm{ps}$ was used. This choice was based on analysis of the data: the calculated AVACF tended to fluctuate around zero for times above $3 \mathrm{ps}$; the AVACF evaluated separately from the two halves of the simulation tended to become uncorrelated for time longer than $5 \mathrm{ps}$; finally, for times longer than 5 ps the calculated integrals tended to fluctuate around stable values. In the MOL AVACF approach, the anthracene angular velocity time series was transformed to the MOL frame, defined by the principal axes of the instantaneous moment of inertia tensor $\mathbf{I}(t)$. This allowed the calculation of rotational diffusion coefficients along individual molecular axes. ${ }^{70,71}$

$$
D_{r, i}=\int_{0}^{\tau_{r}} d t\left\langle\omega_{i}(0) \cdot \omega_{i}(t)\right\rangle, \quad i=x, y, z
$$

as well as their average $D_{r}=\left(D_{r, x}+D_{r, y}+D_{r, z}\right) / 3$. In all the calculation methods above, the statistical errors of the diffusion coefficients were estimated as the standard deviation of the mean of values obtained by dividing the trajectory into ten consecutive fragments.

Rotational diffusion coefficients of anthracene were also derived from correlation functions $C_{1, i}(t)$ for the reorientation of molecular axes, $i=x, y, z$. For a unit vector $n$ $=\left(n_{x}, n_{y}, n_{z}\right)$ rotating with the molecule we define: $C_{1}(t)$ $=\langle n(0) \cdot n(t)\rangle$. For a molecule of high symmetry, such as anthracene, we expect that the principal axes of the moment of inertia and diffusion tensors will coincide. We thus evaluate the time series of the principal axis directions of the anthracene molecule $n_{i}(t), i=x, y, z$, and evaluate their correlation functions $C_{1, i}(t)$. In this case rotational diffusion theory predicts that ${ }^{72,73}$

$$
C_{1, i}(t)=e^{-\left(3 D_{r}-D_{r, i}\right) t}, \quad i=x, y, z .
$$

The calculated $C_{1, i}(t)$ functions were fitted to singleexponential decays and the rotational diffusion coefficients around the individual molecular axes $D_{r, i}$ derived from the exponents. The statistical errors of the exponents derived from the $C_{1, i}(t)$, correlation functions were estimated to $i$ be $\sqrt{2 \tau_{i} / T_{\text {tot }}}$, where $1 / \tau_{i}=3 D_{r}-D_{r, i}$ and $T_{\text {tot }}$ is the total simulation time. ${ }^{69}$ The errors of $D_{r, x}, D_{r, y}$, and $D_{r, z}$ were estimated by error propagation.

\section{E. Calculation of rotational relaxation times}

Experimental methods for studying rotational diffusion measure quantities related to the second order correlation function $C_{2}(t)=\left\langle\left(3\left[(\vec{n}(0) \cdot \vec{n}(t)]^{2}-1\right) / 2\right\rangle,{ }^{74}\right.$ where $\vec{n}$ $=\left(n_{x}, n_{y}, n_{z}\right)$ is a vector rotating with the molecule. For example, the fluorescence anisotropy decay for parallel absorption and emission transition dipoles is given by ${ }^{72,73}$

$$
r(t)=\frac{I_{\|}(t)-I_{\perp}(t)}{I_{\|}(t)+2 I_{\perp}(t)}=\frac{2}{5} C_{2}(t),
$$

where $I_{\|}$and $I_{\perp}$ are the intensities of emitted light with polarization parallel and perpendicular to that of the exciting pulse and $C_{2}(t)$ is the correlation function of the emission dipole axis. Similar expressions hold for methods based on linear dichroism and transient absorption. ${ }^{73}$

Assuming that anthracene reorientations have diffusional character and the molecular axes are also principal axes of the diffusion tensor, for transition dipoles oriented along the $x, y$, or $z$ we expect a biexponential decay for $\mathrm{C}_{2}:{ }^{72,71}$

$$
C_{2, i}(t)=a_{i} e^{-\left(6 D_{r}+2 \Delta\right) t}+b_{i} e^{-\left(6 D_{r}-2 \Delta\right) t}, \quad i=x, y, z,
$$

where $D_{r}$ is the average rotational diffusion coefficient,

$\Delta=\left(D_{r, x}^{2}+D_{r, y}^{2}+D_{r, z}^{2}-D_{r, x} D_{r, y}-D_{r, x} D_{r, z}-D_{r, y} D_{r, z}\right)^{1 / 2}$

is a measure of the anisotropy, and the factors $a_{i}, b_{i}$ are functions of $D_{r, x}, D_{r, y}$, and $D_{r, z}$. The inverses of exponents in the above equation are often called the rotational relaxation or reorientation times

$$
\tau_{1}=\frac{1}{6 D_{r}+2 \Delta}, \quad \tau_{2}=\frac{1}{6 D_{r}-2 \Delta} .
$$

Interestingly, the two exponents are the same for all three axes, only their contributions to $\mathrm{C}_{2}$ differ. If one of the coefficients $a_{i}$ or $b_{i}$ is small, a single-exponential decay of $\mathrm{C}_{2}$ may be observed, even if both times $\tau_{1}$ and $\tau_{2}$ fall into the experimentally accessible time range. ${ }^{73}$

In order to enable direct comparison with experimental data, we have calculated the correlation functions $C_{2, i}(t), i$ $=x, y, z$ for the principal axes of the instantaneous moment of inertial tensor $\mathbf{I}(t)$ of anthracene. In order to minimize ambiguities inherent in fitting noisy data to multi-parameter functions, we used the following fitting procedure to extract time constants and weights for each axis. First we determined the longer relaxation time $\tau_{2}$ and its weight $(1-a)$ from the long-time part of $C_{2, i}(t), t>t_{0}$; the cutoff time $t_{0}$ was based on the average estimate of $\tau_{1}$ from the other methods presented in Table II and was 30 ps for cyclohexane at 296 and $310 \mathrm{~K}, 60 \mathrm{ps}$ for isopropanol at $296 \mathrm{~K}$ and $100 \mathrm{ps}$ for cyclohexane at $280 \mathrm{~K}$. We then determined $\tau_{1}$ by fitting the whole $C_{2, i}(t)$ data to a two-exponential function, $y(t)$ $=a e^{-t / \tau_{1}}+(1-a) e^{-t / \tau_{2}}$, with $a$ and $\tau_{2}$ fixed at the previously determined values. To check the quality of the fit, we also calculated effective correlation times $\tau$ by integrating the $C_{2, i}(t)$ functions, $\tau=a \tau_{1}+(1-a) \tau_{2}$. Within each of the simulation the shortest effective relaxation $\tau_{\min }$ was for $C_{2, y}$; the longest effective relaxation $\tau_{\max }$ was for $C_{2, x}$ in cyclohexane and for $C_{2, z}$ in isopropanol. The times $\tau_{\min }$ and $\tau_{\max }$ were essentially identical to the $\tau_{1}$ and $\tau_{2}$ values, respectively, as obtained from fitting. Only in the $280 \mathrm{~K}$ cyclohexane simulation were significant deviations between these two sets of data found.

We also used the individual diffusion coefficients $D_{r, x}$, $D_{r, y}$, and $D_{r, z}$ determined from fitting the $C_{1}(t)$ correlation functions and the MOL AVACF in conjunction with the 
TABLE I. Average rotational diffusion coefficients of anthracene in cyclohexane (at 280, 296, $310 \mathrm{~K}$ ) and in 2-propanol (at $296 \mathrm{~K}$ ). Diffusion coefficients are in $\operatorname{rad}^{2} / \mathrm{ps}$.

\begin{tabular}{|c|c|c|c|c|c|c|c|c|c|}
\hline \multirow[b]{2}{*}{ Solvent } & \multirow{2}{*}{$\begin{array}{c}\text { Temperature } \\
\text { (K) }\end{array}$} & \multicolumn{4}{|c|}{ Average: $D_{r}$} & \multicolumn{4}{|c|}{ Theory } \\
\hline & & $\mathrm{CM}$ rot. $^{\mathrm{a}}$ & $\mathrm{LAB} A V A C F^{b}$ & MOL AVACF ${ }^{c}$ & $C_{1}{ }^{\mathrm{d}}$ & $\mathrm{sph}$ & $B$ & C & $D$ \\
\hline \multirow[t]{3}{*}{ Cyclohexane } & 280 & $0.004 \pm 0.002$ & $0.005 \pm 0.002$ & $0.010 \pm 0.003$ & $0.003 \pm 0.002$ & 0.0024 & 0.007 & 0.009 & 0.013 \\
\hline & 296 & $0.012 \pm 0.002$ & $0.017 \pm 0.003$ & $0.017 \pm 0.004$ & $0.015 \pm 0.002$ & 0.0033 & 0.009 & 0.013 & 0.018 \\
\hline & 310 & $0.014 \pm 0.004$ & $0.016 \pm 0.003$ & $0.018 \pm 0.003$ & $0.015 \pm 0.002$ & 0.0044 & 0.012 & 0.017 & 0.024 \\
\hline 2-propanol & 296 & $0.006 \pm 0.002$ & $0.007 \pm 0.002$ & $0.011 \pm 0.002$ & $0.007 \pm 0.002$ & 0.0014 & 0.004 & 0.006 & 0.008 \\
\hline
\end{tabular}

${ }^{\mathrm{a}} \mathrm{CM}$ rot. Center of Mass Rotation.

${ }^{\mathrm{b}} \mathrm{LAB}$ AVACF angular velocity autocorrelation function in the laboratory fixed frame.

${ }^{c}$ MOL AVACF angular velocity autocorrelation function in the molecular fixed frame.

${ }^{\mathrm{d}} C_{1}$ autocorrelation functions in the molecular frame; sph sphere with stick boundary condition; $B$ asymmetric ellipsoid (1.0:0.6:0.3) with slip boundary condition; $C$ asymmetric ellipsoid (1.0:0.7:0.3) with slip boundary condition; $D$ asymmetric ellipsoid (1.0:0.7:0.4) with slip boundary condition.

formulas ${ }^{71}$ to obtain separate computational estimates of $\tau_{1}$, $\tau_{2}$ and the weights $a_{i}, b_{i}$. Finally, to correlate our results with theoretical models of rotational diffusion, we used the predicted values of $D_{r, x}, D_{r, y}$, and $D_{r, z}$ for a triaxial ellipsoid under slip conditions ${ }^{23,24}$ to obtain relaxation times based on hydrodynamic theory.

\section{F. Solvent distribution analysis}

The three-dimensional (3D) distribution of the solvent molecules around anthracene was analyzed by repeating the following procedure for each trajectory frame: (a) generating positions of all solvent molecules within $15 \AA$ of the anthracene solute, including image molecules if needed, (b) orienting coordinates of all the atoms to the principal axis system of the instantaneous moment of inertia of the solute, and (c) counting and accumulating the distribution of solvent atoms (or alternatively: solvent centers-of-mass) on a 3D cubic grid of $1 \AA$ side within a range of $\pm 15 \AA$ from the solute center. Comparing the number of counts to that expected for a pure solvent with known molecular volume provides a graphical representation of regions of enhanced or diminished solvent density.

\section{G. Estimates of anthracene size and shape}

We used two methods to estimate anthracene size and shape, based on the van der Waals volume and excluded volume. Using CHARMM van der Waals radii ${ }^{59}$ we obtain molecular dimensions of $11.8,7.8$, and $4.0 \AA$ along the three principal axes. These results indicate that the molecule may be described as a triaxial ellipsoid with axis ratios of 1.0:0.66:0.34; for a symmetric ellipsoid model the average axis ratio of 0.5 would be appropriate. The volume enclosed within the van der Waals radii of anthracene is $223 \AA,^{3}$ as calculated by CHARMM. ${ }^{59}$ A separate estimate of size and shape was made by calculating the average volume free of solvent atoms in the simulations. This was done by a method analogous to the $3 \mathrm{D}$ solvent distribution calculation with the difference being in the use of a finer cubic grid of $0.2 \AA$ over a smaller range of coordinates and counting all solvent atoms, including hydrogens. The excluded volume was calculated by counting the unoccupied bins around the origin. In the isopropanol simulation the molecular dimensions found were 13.1, 9.1, and $4.6 \AA$ along the three principal axes, corresponding to an axis ratio of 1.00:0.69:0.35. In all three cyclohexane trajectories the dimensions were 12.9, 9.0, and $4.9 \AA$, corresponding to an 1.00:0.70:0.38 axis ratio. The excluded volumes from the four simulations fell in the 310 $340 \AA^{3}$ range. Due to the irregular shapes of the cavities occupied by anthracene and the relatively low average number of counts per bin on a $0.2 \AA$ grid, more precise results are difficult to obtain. However, several trends may be found. First, the ellipsoid models of molecular shape are qualitatively similar in excluded volume and van der Waals calculations, with the former having 10\%-20\% larger dimensions, and $\sim 50 \%$ higher volumes. This is in agreement with experimental findings that partial molar volumes of nonpolar solutes are generally significantly higher than their van der Waals volumes. ${ }^{75}$ Second, the largest, and thus most significant, shape change in the excluded volume results is the decrease of the cavity dimension perpendicular to the anthracene plane in isopropanol relative to anthracene.

\section{RESULTS AND DISCUSSION}

\section{A. Average diffusion coefficients}

The calculated rotational diffusion coefficients of anthracene in solution are given in Tables I and II. Four different methods of calculating the average diffusion coefficient $D_{r}$ were used (see Sec. II). Within each simulation all four methods yielded results that agreed within their statistical errors. The mean values of $D_{r}$ from the four calculation methods were $0.006,0.015$, and $0.016 \mathrm{ps}^{-1}$ for cyclohexane solutions at 280,296 , and $310 \mathrm{~K}$, respectively, and $0.008 \mathrm{ps}^{-1}$ for the isopropanol solution at $296 \mathrm{~K}$. These results show general trends in qualitative agreement with experimental observations and theoretical predictions of faster diffusion at higher temperatures and in solvents of lower viscosity. ${ }^{57}$ The simplest model of rotational diffusion is that of a sphere of volume $V$ moving through a medium of viscosity $\eta$ with stick boundary conditions; the diffusion coefficient is then given by the Debye-Stokes-Einstein (DSE) formula $^{13}$ 
TABLE II. Rotational diffusion coefficients of anthracene in cyclohexane (at 280, 296, $310 \mathrm{~K}$ ) and in 2-propanol (at $296 \mathrm{~K}$ ) along individual molecular axis. Diffusion coefficients are in $\mathrm{rad}^{2} / \mathrm{ps}$.

\begin{tabular}{|c|c|c|c|c|c|c|c|c|c|c|c|c|c|}
\hline & \multirow{2}{*}{$\begin{array}{l}\text { Temperature } \\
\text { (K) }\end{array}$} & \multicolumn{6}{|c|}{ Cyclohexane } & \multicolumn{6}{|c|}{ 2-propanol } \\
\hline & & MOL AVACF & $C_{1}{ }^{\mathrm{b}}$ & $A^{\mathrm{c}}$ & $B^{\mathrm{d}}$ & $C^{\mathrm{e}}$ & $D^{\mathrm{f}}$ & MOL AVACF & $C_{1}$ & $A$ & $B$ & C & $D$ \\
\hline & 280 & & & & & & & & & & & & \\
\hline$D_{r, x}$ & & $0.016 \pm 0.009$ & $0.006 \pm 0.002$ & 0.027 & 0.009 & 0.006 & 0.014 & & & & & & \\
\hline$D_{r, y}$ & & $0.007 \pm 0.001$ & $0.001 \pm 0.002$ & 0.004 & 0.002 & 0.002 & 0.004 & & & & & & \\
\hline \multirow[t]{2}{*}{$D_{r, z}$} & & $0.006 \pm 0.002$ & $0.002 \pm 0.002$ & 0.010 & 0.009 & 0.019 & 0.021 & & & & & & \\
\hline & 296 & & & & & & & & & & & & \\
\hline$D_{r, x}$ & & $0.033 \pm 0.005$ & $0.028 \pm 0.003$ & 0.038 & 0.013 & 0.008 & 0.019 & $0.014 \pm 0.002$ & $0.004 \pm 0.002$ & 0.016 & 0.005 & 0.003 & 0.008 \\
\hline$D_{r, y}$ & & $0.008 \pm 0.001$ & $0.005 \pm 0.003$ & 0.006 & 0.003 & 0.003 & 0.006 & $0.004 \pm 0.002$ & $0.003 \pm 0.002$ & 0.002 & 0.001 & 0.001 & 0.003 \\
\hline \multirow[t]{2}{*}{$D_{r, z}$} & & $0.010 \pm 0.003$ & $0.012 \pm 0.003$ & 0.014 & 0.013 & 0.026 & 0.030 & $0.015 \pm 0.002$ & $0.015 \pm 0.002$ & 0.006 & 0.005 & 0.012 & 0.013 \\
\hline & 310 & & & & & & & & & & & & \\
\hline$D_{r, x}$ & & $0.031 \pm 0.008$ & $0.025 \pm 0.003$ & 0.050 & 0.017 & 0.011 & 0.025 & & & & & & \\
\hline$D_{r, y}$ & & $0.007 \pm 0.002$ & $0.004 \pm 0.003$ & 0.007 & 0.004 & 0.004 & 0.008 & & & & & & \\
\hline$D_{r, z}$ & & $0.016 \pm 0.004$ & $0.017 \pm 0.003$ & 0.019 & 0.017 & 0.035 & 0.039 & & & & & & \\
\hline
\end{tabular}

${ }^{\mathrm{a} M O L}$ AVACF angular velocity autocorrelation function in the molecular fixed frame.

${ }^{\mathrm{b}} C_{1}$ autocorrelation functions of molecular axis.

${ }^{\mathrm{c}} A$ slip hydrodynamics, axis ratio $1: 0.6: 0.4$.

${ }^{\mathrm{d}} B$ slip hydrodynamics, axis ratio 1.0:0.6:0.3.

${ }^{\mathrm{e}} \mathrm{C}$ slip hydrodynamics, axis ratio $1.0: 0.7: 0.3$.

${ }^{\mathrm{f}} D$ slip hydrodynamics, axis ratio 1.0:0.7:0.4.

$$
D_{r}=\frac{k T}{6 \eta V} .
$$

Setting $V=223 \AA^{3}$ - the volume enclosed within the van der Waals surface of anthracene, and the viscosity $\eta$ to $1.2,0.9$, 0.7 , and $2.1 \mathrm{cP}$ (Ref. 76) — corresponding to pure cyclohexane at 280,296 , and $310 \mathrm{~K}$ and pure isopropanol at $296 \mathrm{~K}$, respectively, we obtain the respective $D_{r}$ values of 0.0024 , $0.0033,0.0044$, and $0.0014 \mathrm{ps}^{-1}$. The relative values of the computed diffusion coefficients $D_{r}$ are approximately reproduced by these results. However, the absolute values of the calculated diffusion coefficients are about 3-5 times higher than the above DSE treatment. Similar effects have been found in experimental studies. ${ }^{77,78}$ These deviations may be explained by two main factors-diffusion anisotropy and deviation of boundary conditions from stick type.

Under stick conditions the effects of anisotropy are predicted to be small. Using Perrin's formulas, ${ }^{19,20}$ we obtain a shape factor (equal to the ratio of the rotational friction coefficient to that of a sphere of equal volume) of $F=1.2$ for a prolate symmetric ellipsoid with axis ratio 0.5 , and $F=1.3$ for an asymmetric ellipsoid with ratio of axes of 1.0:0.66:0.34. Applying these correction factors would decrease the DSE diffusion coefficients by $20 \%-30 \%$, moving them further away from the calculated results.

A number of simulations and experimental studies suggest that slip boundary conditions may be appropriate for many organic molecules in solution. ${ }^{43,79-81}$ Faster diffusion rates are expected under slip conditions; ${ }^{79,80}$ e.g., a symmetric ellipsoid should undergo free rotation around its symmetry axis, since such motion displaces no solvent. Using the results of Youngren and Acrivos ${ }^{23}$ as corrected by Hochstrasser and Sension, ${ }^{24}$ we obtain diffusion coefficients in much better agreement with the calculated results. Using the same solvent viscosities as described above and coefficients from Table I of (Ref. 24) for an ellipsoid with volume $V$ $=223 \AA^{3}$ and axis ratios 1.0:0.6:0.3, we obtain diffusion coefficients $D_{r}$ of $0.07,0.09,0.012 \mathrm{ps}^{-1}$ for anthracene in cyclohexane at 280, 296, and $310 \mathrm{~K}$, respectively, and $0.004 \mathrm{ps}^{-1}$ in isopropanol at $296 \mathrm{~K}$. The corresponding values for axis ratios of 1.0:0.7:0.3 are 0.009, 0.013, 0.017 and $0.006 \mathrm{ps}^{-1}$, respectively. Both these sets of values agree with the corresponding simulation results within the statistical errors.

Our calculated average diffusion coefficients are thus consistent with hydrodynamic theory predictions under slip conditions with reasonable assumptions of anthracene shape and size. Comparable agreement has been found for experimental results for similar systems. ${ }^{33,45,56}$ This suggests that our results are reasonable. We may thus use the wealth of information contained in the trajectories to calculate quantities that are difficult to measure experimentally but provide valuable insight into the molecular details of the observed processes. We will focus on three aspects-diffusion anisotropy, analysis of solvent structure, and details of solute and solvent motions.

\section{B. Anisotropy}

The molecular axes of anthracene are shown in Fig. 1. The calculated rotational diffusion coefficients of anthracene $D_{r}, x, D_{r, y}, D_{r, z}$ around its three molecular axes are presented in Table II. Calculations were carried out using two independent approaches - by fitting the first-order correlation functions $C_{1, i}(t), i=x, y, z$ describing the reorientation of the axes, and by integrating the correlation functions of the CM angular velocity components in the molecular frame (see Sec. II). The corresponding rotational diffusion coefficients 


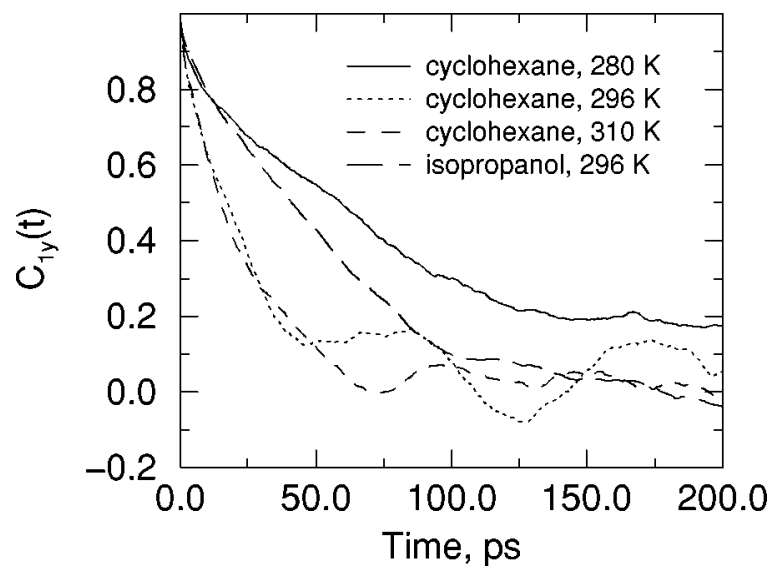

FIG. 2. $C_{1}$ correlation functions for reorientation of the molecular $y$ axis of anthracene in cyclohexane at 280, 296, and $310 \mathrm{~K}$ and in 2-propanol at 296 K.

calculated by these two methods agreed within their uncertainties in most cases. The only exceptions were $D_{y}$ in cyclohexane at $280 \mathrm{~K}$ and $D_{x}$ in isopropanol, for which the two calculated values differed by up to twice the sum of errors. These deviations are probably related to the relatively poor sampling of orientations due to slow rotation in the simulations in question (see Sec. II). Examples of the calculated $C_{1}$ correlation functions for axis reorientation and of anthracene AVACFs are presented in Figs. 2 and 3.

The rotational diffusion of anthracene found in the simulations is highly anisotropic, with ratios of greatest to smallest diffusion coefficients ranging from $6: 1$ to $4: 1$ (Table II). Additionally, the diffusion coefficients undergo nonuniform changes with temperature and solvent type. In cyclohexane there is a systematic tendency for fastest rotation around the $x$ axis. Rotations around $y$ and $z$ occur at similar rates in the $280 \mathrm{~K}$ cyclohexane simulation, but $D_{z}$ becomes markedly larger than $D_{y}$ at 296 and $310 \mathrm{~K}$. Comparison of the cyclohexane and isopropanol $296 \mathrm{~K}$ simulations also shows a highly nonuniform change in diffusion coefficients. $D_{x}$ becomes significantly lower in isopropanol, $D_{z}$ is slightly in-

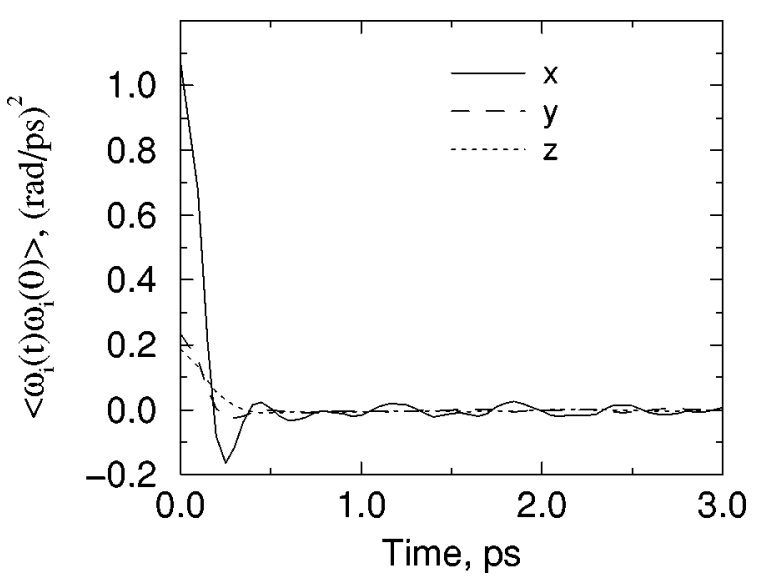

FIG. 3. Autocorrelation functions of the components of the center-of-mass angular velocity of anthracene in cyclohexane at $296 \mathrm{~K}$ in the moleculefixed axis system. creased, while $D_{y}$ remains practically the same as in cyclohexane, within the uncertainties. In isopropanol the pattern of rotational diffusion changes qualitatively, with motion around $z$ becoming at least as fast as (based on AVACF) or faster than (based on $C_{1}$ ) around $x$.

For an asymmetric ellipsoid under slip conditions, we would expect similar diffusion rates around $x$ and $z$ and a $D_{r, z} / D_{r, y}$ ratio of about 4 for an axis ratio of 1.0:0.6:0.3, and fastest diffusion around $z$ with $D_{r, z} / D_{r, y}$ of about 5-7 for axis ratios of 1.0:0.7:0.3 and 1.0:0.7:0.4 (see Table II). ${ }^{24}$ The isopropanol simulation results fall somewhere in between the latter two theoretical predictions. The cyclohexane result of fastest rotational diffusion around $x$ is in qualitative disagreement with hydrodynamic theory predictions for ellipsoids with axis ratios consistent with the shape of the anthracene solute. Interestingly, $D_{r, x}$ becomes the largest diffusion coefficient for an axis ratio that is only slightly different1.0:0.6:0.4 (Table II). It thus seems that anthracene falls into a boundary region between prolate- and oblatelike shapes, and small differences in assumed shapes lead to markedly different values of $D_{r, x}, D_{r, y}$, and $D_{r, z}$. The average diffusion coefficient $D_{r}$ does not exhibit such sensitivity.

\section{Solvent structure}

Some of the nonuniform changes of diffusion patterns with temperature and solvent may be rationalized by analyzing the solvent distributions around anthracene in the different simulations. Due to the large size and relatively complex structure of the solute, only limited information could be extracted from atom-atom pair correlation functions. For this reason we focus on the more informative 3D solvent distributions calculated in the solute-fixed frame (see Sec. II for details). The three-dimensional solvent distributions provide a powerful description of solvation and reveal several interesting features, presented in Figs. 4-11.

The cyclohexane results show the presence of a well defined first solvation shell and two additional, less defined shells around anthracene (Figs. 4-8). Further, a definite solvent structure appears in the solute molecular plane, with eight well-resolved density peaks. At $280 \mathrm{~K}$ the solvation structure is more resolved than at the higher temperatures, with better separation of density peaks in the first solvation shell, and less smearing out of the density in the second and third solvation shells (Fig. 4-7). The more rigid solvent structure in the solute plane would explain the significant slowing down of the rotation around the $z$ axis at $280 \mathrm{~K}$. Especially interesting are the changes with temperature in the "solvent cap" above and below the anthracene plane. At the higher temperatures-296 and $310 \mathrm{~K}$, the solvent density in this region is concentrated at the center of the solute. At $280 \mathrm{~K}$ we can see well resolved solvent density peaks above and below the anthracene molecular plane; if the anthracene molecule were modeled as a rectangle, the increased solvent density would be over the rectangle corners (Figs. 5 and 7). This pattern of solvation could be expected to hinder rotation around the $x$ and $y$ axes. The slowing down of rotation around $x$ at $280 \mathrm{~K}$ found in the simulations is consistent with this picture. However, the simulations indicate that the rota- 


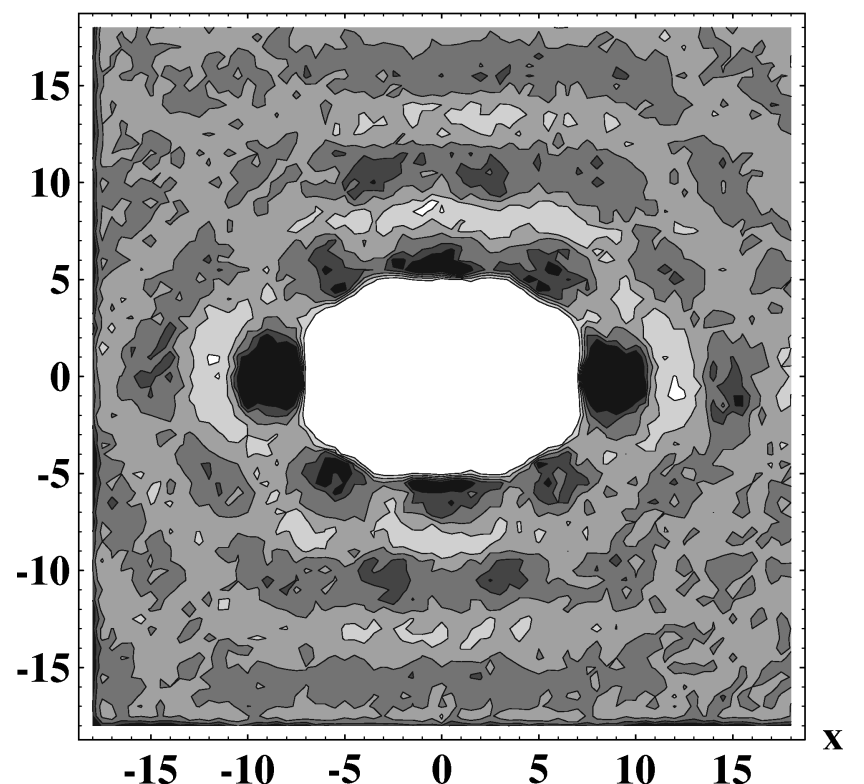

FIG. 4. Distribution of cyclohexane carbons around anthracene: slice in $X Y$ plane containing solute, $280 \mathrm{~K}$. Grayscale coloring proportional to ratio $\rho^{*}$ of local to average solvent density: white $-\rho^{*}<0.5$, black $-\rho^{*}>2.0$.

tion around $y$ remains approximately constant, at all studied temperatures and in both solvents (Table II). Thus it appears that the rotation around $y$, which displaces the most solvent, depends only weakly on the details of the solvent structure. The solvent structure in the $310 \mathrm{~K}$ simulation is very similar to the $296 \mathrm{~K}$ results, and is not shown,

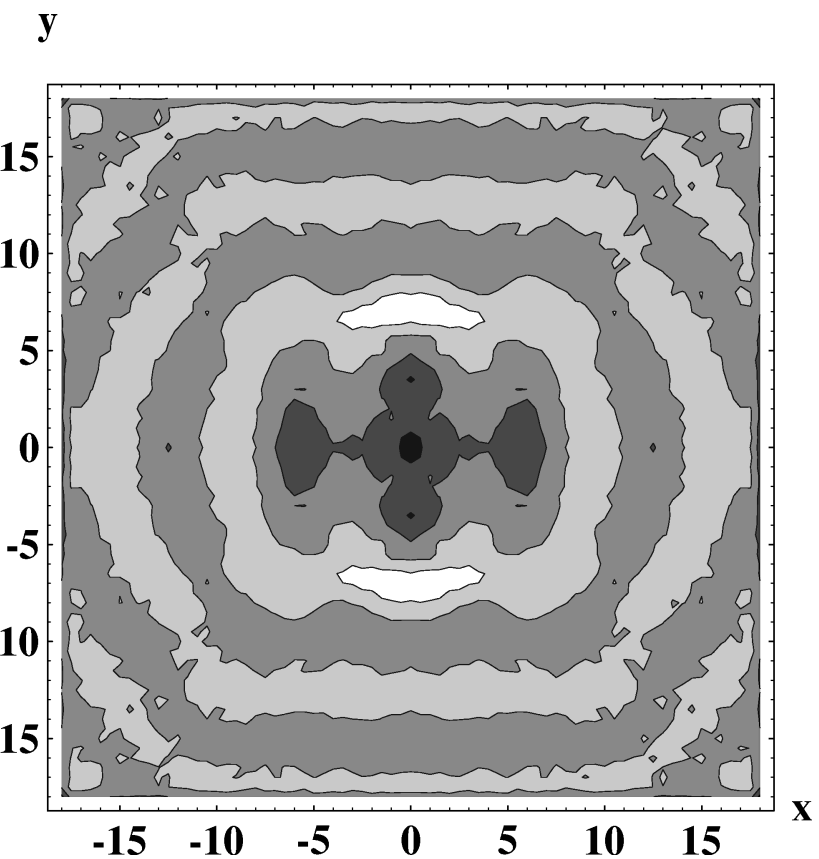

FIG. 5. Distribution of cyclohexane carbons around anthracene: slice at 4.5 $\AA$ above the $X Y$ plane containing the solute, $D_{2 h}$ symmetry averaged, 280 K. Coloring as in Fig. 4.

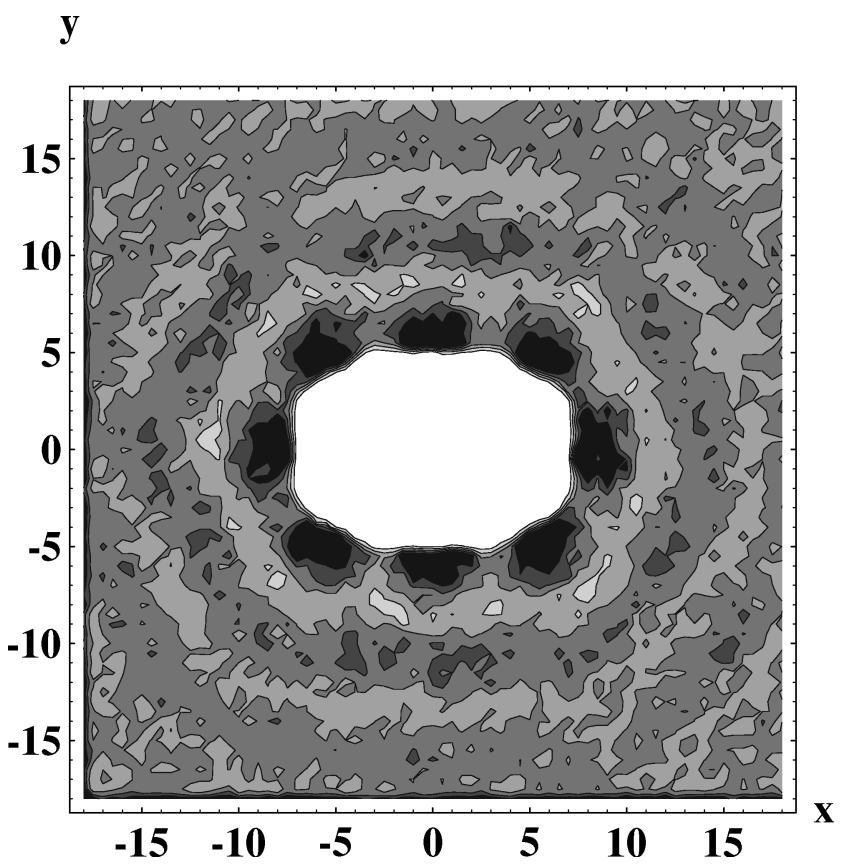

FIG. 6. Distribution of cyclohexane carbons around anthracene: slice in $X Y$ plane, 296 K. Coloring as in Fig. 4.

The solvation of anthracene by isopropanol is markedly different than that by cyclohexane. As seen using molecular graphics, ${ }^{82}$ the isopropanol molecules interact strongly with each other, tending to form hydrogen-bonded chains. This explains why the structure of isopropanol is less perturbed by the presence of anthracene. In the $296 \mathrm{~K}$ isopropanol simulation only one solvation shell is well resolved (Figs. 9-11). Eight solvent peaks may be seen in the solute molecular

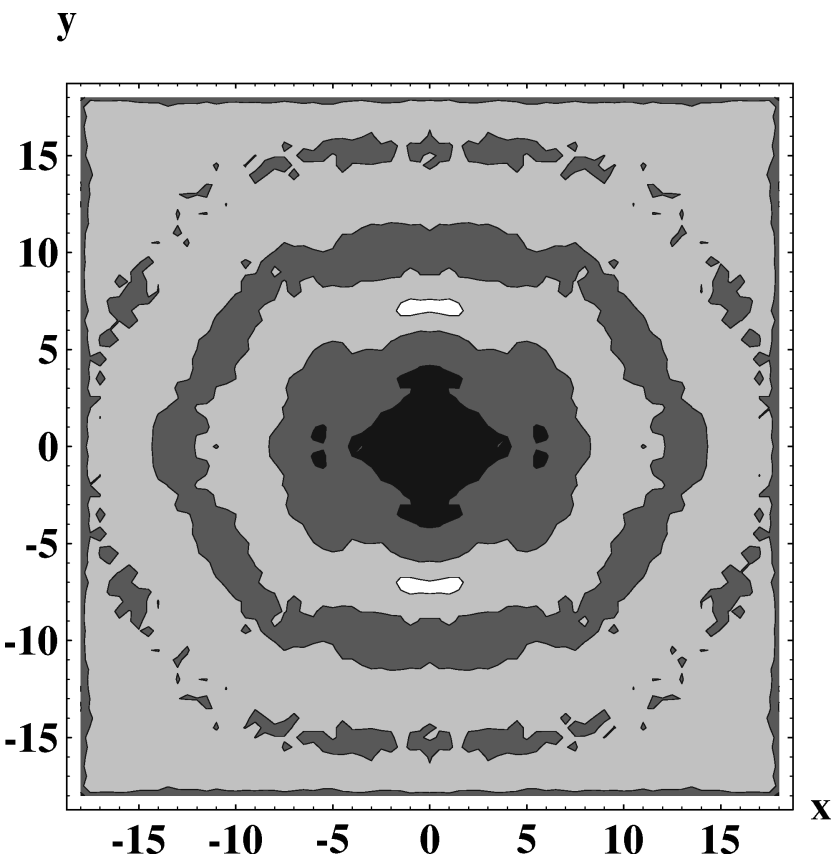

FIG. 7. Distribution of cyclohexane carbons around anthracene: a slice at $4.5 \AA$ above the $X Y$ plane, $D_{2 h}$ symmetry averaged, $296 \mathrm{~K}$. Coloring as in Fig. 4. 


\section{$\mathbf{Z}$}

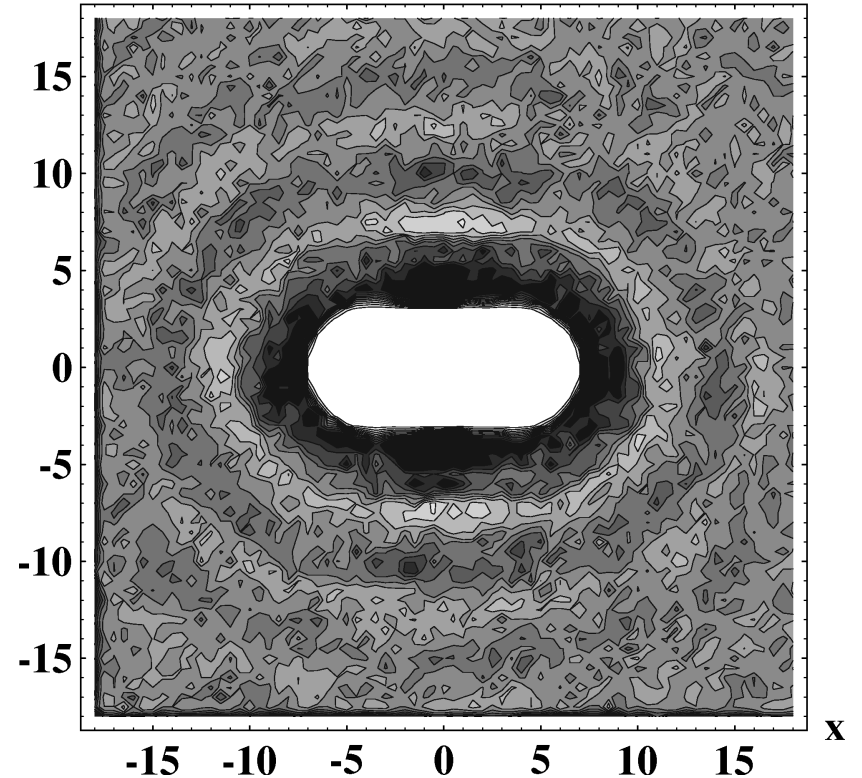

FIG. 8. Distribution of cyclohexane carbons around anthracene: slice in $X Z$ plane perpendicular to solute, $296 \mathrm{~K}$. Coloring as in Fig. 4.

plane, which are less well defined than in cyclohexane. This more "loose" structure of the solvation shell in the anthracene plane may explain the faster diffusion rate around the $z$ axis in isopropanol relative to cyclohexane. An interesting picture emerges when carbon and oxygen atom distributions above the anthracene molecular plane are analyzed separately (Figs. 9 and 10). While the carbon distribution yields a simple, relatively smeared out first solvation shell, the oxygen distribution is split into two components,

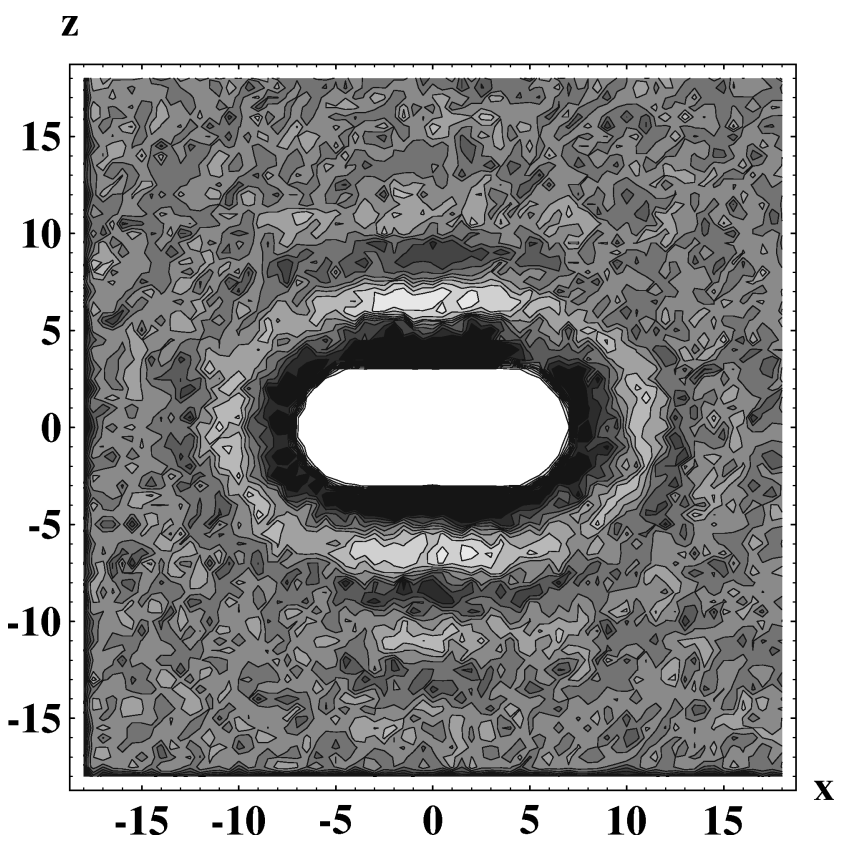

FIG. 10. Distribution of 2-propanol carbons around anthracene: slice in $X Z$ plane, 296 K. Coloring as in Fig. 4.

corresponding to isopropanol molecules oriented toward and away from the solute (Figs. 10 and 11). It is the former component in which the closest contacts between isopropanol molecules and anthracene carbons occur. The presence of this component may explain the preferential slowing down of the rotation around the $x$ and $y$ axis in isopropanol as compared to cyclohexane. Preferential interactions of hydroxyl oxygens with aromatic rings have been observed previously. ${ }^{83}$

\section{$\mathbf{y}$}

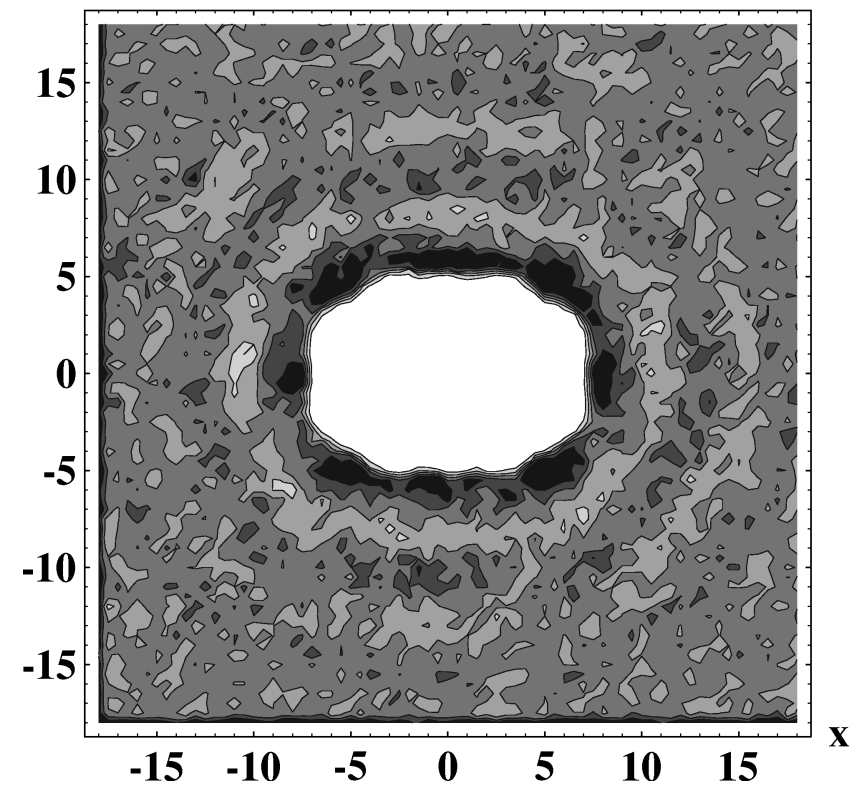

FIG. 9. Distribution of 2-propanol carbons around anthracene: slice in $X Y$ plane, 296 K. Coloring as in Fig. 4.

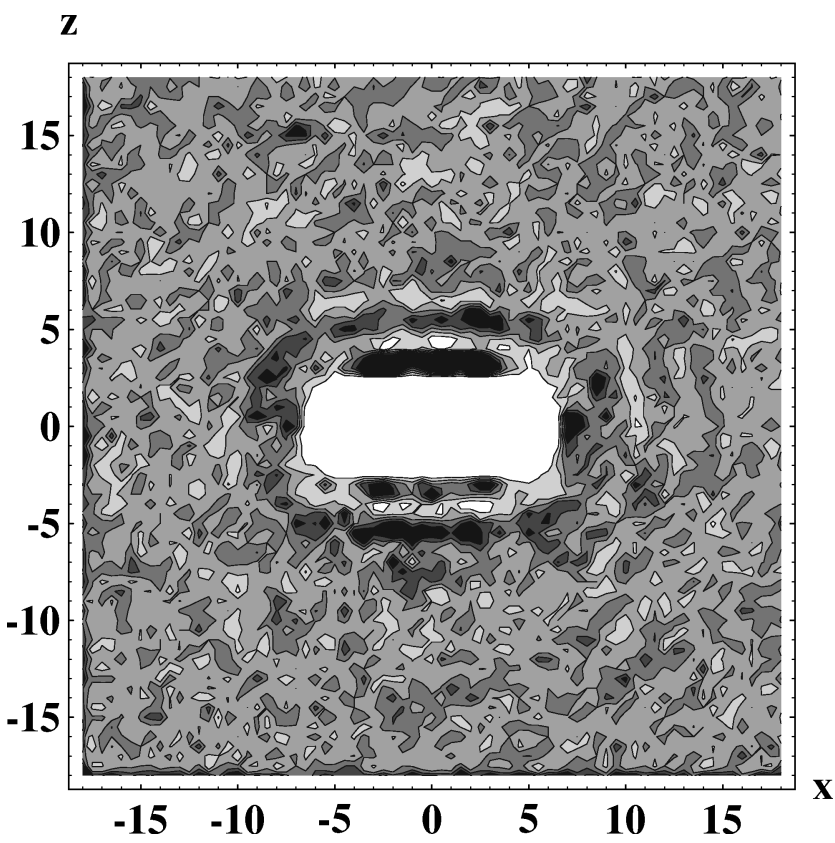

FIG. 11. Distribution of 2-propanol oxygens around anthracene: slice in $X Z$ plane, 296 K. Coloring as in Fig. 4. 
TABLE III. Reorientation times of anthracene in cyclohexane (at 280, 296, $310 \mathrm{~K}$ ) and in 2-propanol (at $296 \mathrm{~K}$ ). Reorientation times are in ps.

\begin{tabular}{|c|c|c|c|c|c|c|c|c|c|c|c|c|}
\hline & \multicolumn{9}{|c|}{ Cyclohexane } & \multirow{2}{*}{\multicolumn{3}{|c|}{$\frac{\text { 2-propanol }}{296 \mathrm{~K}}$}} \\
\hline & \multicolumn{3}{|c|}{$280 \mathrm{~K}$} & \multicolumn{3}{|c|}{$296 \mathrm{~K}$} & \multicolumn{3}{|c|}{$310 \mathrm{~K}$} & & & \\
\hline & $\tau_{1}$ & $\tau_{2}$ & $a^{\mathrm{a}}$ & $\tau_{1}$ & $\tau_{2}$ & $a^{\mathrm{a}}$ & $\tau_{1}$ & $\tau_{2}$ & $a^{\mathrm{a}}$ & $\tau_{1}$ & $\tau_{2}$ & $a^{\mathrm{a}}$ \\
\hline MOL VACF & $13 \pm 4$ & $26 \pm 8$ & $0.0,0.7,0.8$ & $7 \pm 1$ & $18 \pm 5$ & $0.0,0.8,0.7$ & $7 \pm 1$ & $15 \pm 4$ & $0.0,0.9,0.6$ & $11 \pm 1$ & $22 \pm 4$ & $0.3,1.0,0.2$ \\
\hline$C_{1}$ & $36 \pm 12$ & $113 \pm 30$ & $0.0,0.8,0.7$ & $8 \pm 1$ & $20 \pm 5$ & $0.0,0.9,0.6$ & $8 \pm 1$ & $18 \pm 4$ & $0.1,1.0,0.4$ & $15 \pm 2$ & $48 \pm 20$ & $0.7,0.8,0.0$ \\
\hline$C_{2, y}^{\mathrm{b}}$ & 71.0 & 143.0 & & 9.0 & 22.0 & & 7.0 & 16.0 & & 16.0 & 32.0 & \\
\hline$A^{\mathrm{c}, y}$ & 8 & 25 & $0.9,0.2,0.4$ & 6 & 17 & $0.9,0.2,0.4$ & 4 & 14 & $0.9,0.2,0.4$ & 14 & 43 & $0.9,0.1,0.4$ \\
\hline$B^{\mathrm{d}}$ & 18 & 38 & $0.7,0.0,0.7$ & 13 & 26 & $0.7,0.0,0.7$ & 10 & 20 & $0.7,0.0,0.7$ & 33 & 71 & $0.7,0.0,0.7$ \\
\hline$C^{\mathrm{e}}$ & 12 & 43 & $0.3,0.2,0.9$ & 9 & 31 & $0.3,0.2,0.9$ & 6 & 23 & $0.4,0.2,0.9$ & 19 & 85 & $0.3,0.2,0.9$ \\
\hline$D^{\mathrm{f}}$ & 9 & 21 & $0.5,0.0,0.9$ & 7 & 15 & $0.5,0.1,0.9$ & 5 & 11 & $0.5,0.1,0.9$ & 15 & 33 & $0.5,0.1 .0 .9$ \\
\hline
\end{tabular}

${ }^{\mathrm{a} C a l c u l a t e d}$ weights of $\tau_{1}$ exponent in $C_{2 x}, C_{2 y}, C_{2 z}$.

${ }^{\mathrm{b}} C_{2}(t)=a \exp \left(-t / \tau_{1}\right)+(1-a) \exp \left(-t / \tau_{2}\right)$.

${ }^{\mathrm{c}} A=$ slip hydrodynamics, axis ratio 1:0.6:0.4.

${ }^{\mathrm{d}} B=$ slip hydrodynamics, axis ratio $1.0: 0.6: 0.3$.

${ }^{\mathrm{e}} \mathrm{C}=$ slip hydrodynamics, axis ratio 1.0:0.7:0.3.

${ }^{\mathrm{f}} D=$ slip hydrodynamics, axis ratio 1.0:0.7:0.4.

\section{Comparison with experiment}

Table III characterizes the $C_{2}$ correlation functions derived directly and indirectly from our simulations and from hydrodynamic models. These results - values of the two relaxation times $\tau_{1}$ and $\tau_{2}$ as well as weights of the two exponents in $C_{2}(t)$-are directly comparable to experimental data (see Sec. II). The $C_{2, y}(t)$ decays obtained from the simulations are shown in Fig. 12.

Our simulation results predict $\tau_{1}$ values of $13-71,7-9$, and 7-8 ps for anthracene in cyclohexane at 280,296 , and $310 \mathrm{~K}$, and $\tau_{2}$ of $26-143,18-22$, and $15-18$ ps under the same conditions. For anthracene in isopropanol at $296 \mathrm{~K}$ we obtain $\tau_{1}$ of $11-16$ ps and $\tau_{2}$ of $22-48$ ps. Generally, the sets of different results obtained for a given system based on VACF, $C_{1, i}(t)$ and $C_{2, i}(t)$ are internally consistent, with results obtained by different methods agreeing within their statistical errors. The agreement between results obtained by different methods is best for cyclohexane at 296 and $310 \mathrm{~K}$, where the relaxation times are shortest and thus the best statistics are obtained. The widest distribution of calculated val-

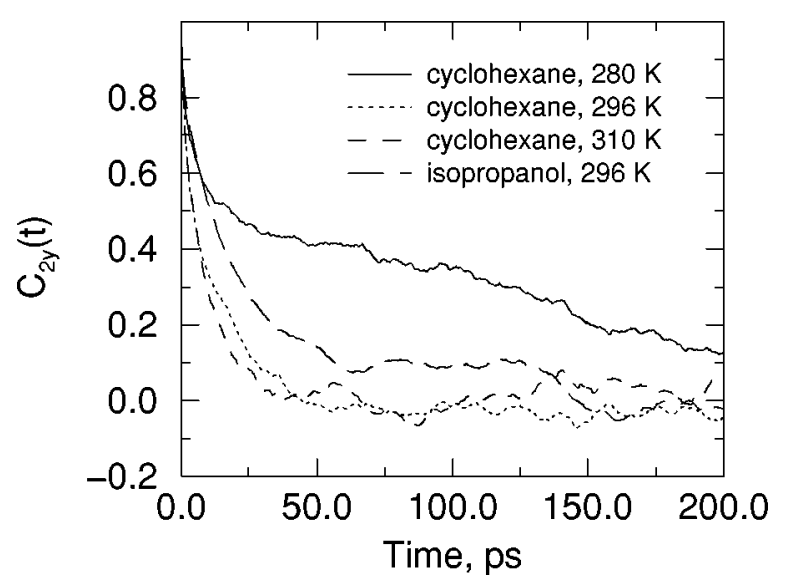

FIG. 12. $C_{2}$ correlation functions for reorientation of the molecular $y$ axis of anthracene in cyclohexane at 280, 296, and $310 \mathrm{~K}$, and in 2-propanol at 296 K. ues is for cyclohexane at $280 \mathrm{~K}$, where the relaxation times are longest and the statistics are poorest. Various effects contribute to make reliable computations of the rotational relaxation times difficult. In the indirect approaches based on Eq. (6), $\tau_{1}$ and $\tau_{2}$ are obtained from $D_{r}$ and $\Delta$, which are already derivative quantities in terms of the basic parameters $D_{r, x}$, $D_{r, y}, D_{r, z}$. The reliability of $\tau_{2}$ values is further limited by the fact that they are obtained from a difference of two positive quantities $D_{r}$ and $\Delta$. Extracting the two relaxation times by direct fitting of $C_{2, i}(t)$ is also complicated by the noisiness of the data and the fact that the weight of one of the exponentials is often small.

The range covered by the simulation results overlaps with the different hydrodynamic theory values obtained under reasonable assumptions about anthracene shape and size. For the three cyclohexane simulations, the MOL AVACF results correlate very well with slip hydrodynamic predictions for a triaxial ellipsoid with volume $223 \AA^{3}$ and axis ratios of either 1:0:0.7:0.3 or 1.0:0.6:0.4, the latter being the shape which also best reproduced the calculated diffusion anisotropies. For the isopropanol simulation the calculated reorientation times agree with slip hydrodynamic results for axis ratios of either 1.0:0.6:0.4 or 1.0:0.7:0.4, which bracket the shape found from excluded volume analysis (1.00:0.69:0.35, see Sec. II). Our simulation results are consistent with the finding of faster rotational diffusion in alcohols compared to alkane solvents of similar viscosity. ${ }^{57}$ As can be seen from Table III, both simulations and hydrodynamic theory predict that the term with the shorter relaxation time $\tau_{1}$ will be a major component of $C_{2, y}(t)$, with weights in the 0.6-1.0 range. For $C_{2, x}(t)$ the opposite is predicted, with major contributions from the $\tau_{2}$ term.

Most available experimental data, including our measurements, are for the reorientation of the short in-plane axis of anthracene, $y$ in our notation (Fig. 1), which is the direction of the $S_{0} \rightarrow S_{1}$ transition dipole. ${ }^{33,45,56}$ Fluorescence depolarization measurements of rotational reorientation times shown in Table IV. Since it was not possible to discern more 
TABLE IV. Measured and simulated reorientation times of anthracene in cyclohexane and in 2-propanol at several different temperatures. Reorientational times are in ps.

\begin{tabular}{cccc}
\hline \hline Solvent & $\begin{array}{c}\text { Temperature } \\
(\mathrm{K})\end{array}$ & $\begin{array}{c}\text { Measured } \\
\left(\tau_{1}\right)\end{array}$ & $\begin{array}{c}\text { Simulated } \\
\left(\tau_{1}\right)\end{array}$ \\
\hline Cyclohexane & 280 & & $13-71$ \\
& 283 & 12 & \\
& 293 & 8 & $7-9$ \\
& 296 & & $7-8$ \\
2-propanol & 310 & & \\
& 263 & 36 & \\
& 286 & 21 & $11-16$ \\
\hline \hline
\end{tabular}

than one component in the fluorescence anisotropy decay, these values should be regarded as weighted averages of the underlying reorientational times. The rotational correlation times of 8 ps at $296 \mathrm{~K}$ and $12 \mathrm{ps}$ at $280 \mathrm{~K}$ for anthracene in cyclohexane agree most closely with the predicted values of $\tau_{1}$ in Table III, consistent with the high amplitude for the shorter relaxation time for reorientation of the emission axis $(y)$. The closest agreement is found with the correlation times calculated by the AVACF method. Similarly, for anthracene in isopropanol at $296 \mathrm{~K}$ the measured rotational correlation time $(14 \mathrm{ps})$ is closest to the predicted $\tau_{1}$ reorientation time.

The experimental results are in good agreement with DSE theory under slip boundary conditions. Figure 13 shows the dependence of the measured rotational reorientation time on the ratio $\eta / T$ of viscosity to temperature. For comparison, the slip hydrodynamic predictions for the $\eta / T$ dependence of $\tau_{1}$ are also shown in Fig. 13. The experimentally determined

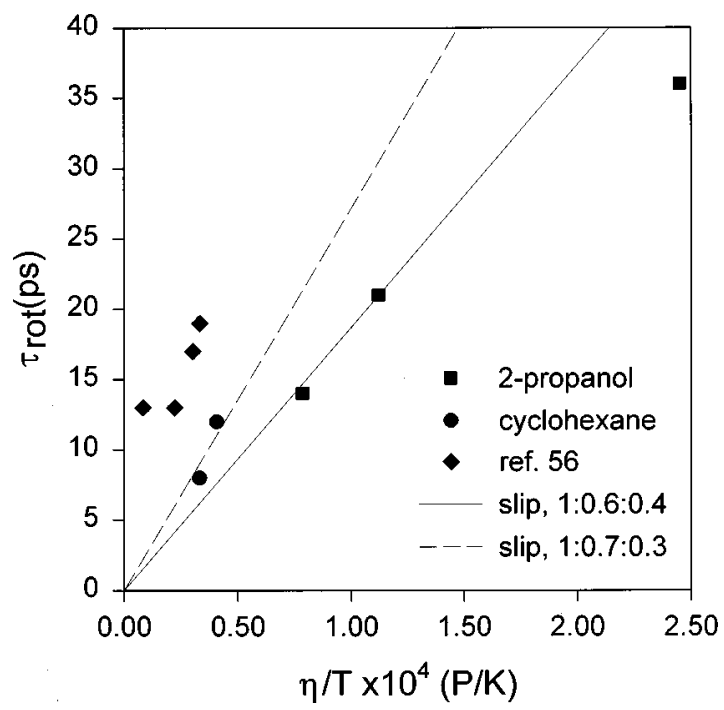

FIG. 13. Dependence of the measured rotation time of anthracene on the ratio of viscosity to temperature. Rotation times are shown for anthracene in 2-propanol ( $\boldsymbol{\square}$, this work) and cyclohexane (, this work). Rotation times are also shown for anthracene in $\mathrm{C}_{3} \mathrm{D}_{6} \mathrm{O}, \mathrm{C}_{6} \mathrm{D}_{6}, \mathrm{C}_{2} \mathrm{Cl}_{4}$, and $\mathrm{CCl}_{4}(\diamond, 56)$. The slip boundary condition predictions for $\tau_{1}$ are shown for comparison for two axial ratios: 1.0:0.6:0.4 (solid line) and 1.0:0.7:0.3 (dashed line). rotational relaxation times agree well with slip predictions for axial ratios 1:0.6:0.4 (solid line) and 1:0.7:0.3 (dashed line) and a volume of $223 \AA^{3} .{ }^{23,24}$ Although the data shown in Fig. 12 roughly follow slip boundary conditions, a single parameter (the viscosity) clearly cannot account completely for the observed solvent dependence. A dependence of the apparent friction on the nature of the solvent has been noted previously. ${ }^{57}$ Hence, a consistent viscosity dependence observed for rotational relaxation in a series of like solvents may not generalize to dissimilar solvents. For example, the slight difference in the apparent viscosity dependence for isopropanol and cyclohexane is consistent with previous observations of a lower friction coefficient for nonpolar solutes in alcohols compared to alkanes. ${ }^{57}$ The relatively higher friction and longer reorientation times at a given viscosity found previously for anthracene in acetone- $d_{6}$, benzene- $d_{6}$, ethylene chloride, and carbon tetrachloride ${ }^{56}$ may also reflect specific solute-solvent interactions or the solvent structure surrounding the solute. Such factors may affect the microscopic friction in ways that are not reflected in the viscosity, which is a property of the pure solvent.

Dorfmuller et al..$^{33}$ measured both the long $(x)$ and short (y) axis reorientation times for anthracene at room temperature, each in a different solvent. The reduced reorientation time $\tau_{1} T / \eta$ around the $y$ axis for anthracene in paraffin was from $1.66 \times 10^{3}$ to $1.85 \times 10^{3}$ ( $\mathrm{ps} \mathrm{K} / \mathrm{cP}$ ), ${ }^{33}$ which is nearly identical to the dependence observed here for anthracene in isopropanol, even though the rotational time in paraffin is three orders of magnitude longer. Additionally, these authors found that the reduced reorientation time $\tau / \eta$ of the $x$ axis was $\sim 3.5$ times that for $y$. This is in qualitative agreement with our $296 \mathrm{~K}$ simulation results - in cyclohexane and isopropanol $\tau_{2} / \tau_{1}$ ratios were between 2 and 3 .

\section{E. General features of anthracene motion}

Some general observations about the motion of the anthracene molecule may be made based on the simulation results. Thus anthracene undergoes free motion on subpicosecond time scales, its translations and rotations are randomized over a period of several picoseconds, and it performs diffusive-type motion over hundreds of picoseconds. On the shortest time scales of $0.1-0.3$ the $C_{1}(t)$ and $C_{2}(t)$ correlation functions for molecular axis reorientation have Gaussian shape, and may be fitted by $e^{-k T t^{2} / 2 I}$ with $I$ the moment of inertia, as expected for a free rotor. Linear variation of CM displacement and rotation with time persists for longer times - up to several picoseconds. In our model anthracene is flexible, with only $\mathrm{C}-\mathrm{H}$ stretch vibrations eliminated by SHAKE constraints. Although the data for $C_{1}$ and $C_{2}$ calculations are sampled every $0.05 \mathrm{ps}$, which is comparable with the period of the slowest anthracene oscillation, we do not see any vibrational effects in the correlation functions. Both the angular velocity and velocity autocorrelation functions (not shown) decay essentially to zero within $2-3$ ps. This is thus the period during which the translation and rotation of the solute are randomized through multiple collisions with solvent. Except for the subpicosecond time scales, the $C_{1}$ 
correlation functions for reorientations of the molecular axes of anthracene may be reasonably well fitted by single exponential decays, especially within the first 100-200 ps. For longer times some deviations are found, especially in the simulations with smaller mobility. However, since in these situations the data sampling deteriorates, reliable conclusions cannot be drawn from this behavior. The square of the CM displacement is linear with time for up to 300-400 ps (not shown), while the square of the angle of CM rotation is approximately linear with time for up to 50-100 ps. Thus, the motion of anthracene may be described as diffusional on time scales of tens to hundreds of picoseconds. The smaller region of diffusive behavior seen in the $\mathrm{CM}$ rotation may be due to the limitation of the formula to small angles- the average will not be correct if angles above $\pi$ are encountered.

\section{CONCLUSIONS}

We have employed a two-pronged approach, using both fluorescence anisotropy decay measurements and molecular dynamics simulations, to investigate the rotational diffusion of anthracene as a function of temperature and solvent type. The fluorescence measurements yielded rotational reorientation times for the $S_{0} \rightarrow S_{1}$ transition dipole (short axis, $y$ in our notation). Molecular dynamics simulations were used to calculate the average rotational diffusion coefficients, diffusion coefficients and reorientation correlation functions for the individual molecular axes, as well as the average distribution of solvent molecules around the anthracene solute. The calculated time constants for reorientation of the short in-plane axis were 7-9 and $11-16 \mathrm{ps}$ at $296 \mathrm{~K}$ in cyclohexane and 2-propanol, respectively, in excellent agreement with corresponding fluorescence depolarization measurements of 8 and 14 ps.

The calculated average rotational diffusion coefficients, as well as both calculated and measured rotational reorientation times varied approximately in accord with DebyeStokes-Einstein theory. Their magnitudes were close to values predicted for an ellipsoid of shape and size equivalent to an anthracene molecule under slip conditions, and exhibited predictable variation with external conditions-increasing with temperature and decreasing with solvent viscosity. However, it is clear that a single parameter-the viscositycannot account completely for the observed solvent dependence in the simulated and measured reorientation times, and that details of the microscopic solute-solvent interactions have to be considered.

Further, detailed analysis of the calculated rotational diffusion coefficients for the individual molecular axes gave a more complex picture of solute diffusion. The diffusion was highly anisotropic and changes in temperature and solvent type led to nonuniform variation of the diffusion coefficients. The nature of these changes could be rationalized based on analysis of variation of solvation patterns with temperature and solvent. In the case of cyclohexane, which exhibits weak self-interactions, the main effect seen was the appearance of more and better defined solvent density peaks around the solute at lower temperatures. In isopropanol, which is strongly self-interacting and forms hydrogen-bonded chains, a close contact between the hydroxyl oxygen and the anthracene plane was detected, which does not occur for either isopropanol or cyclohexane carbons. Both these effects correlate with corresponding changes in diffusion anisotropy.

Our results suggest that the general agreement of the average rotational correlation times with simple theories, often observed for probe molecules such as anthracene, ${ }^{57}$ may disguise interesting and informative variations in the rotational diffusion coefficients for specific molecular axes. These variations may reflect specific solute-solvent interactions and the microscopic effects of solvent structure. Our results should be a helpful reference for analyzing experimental and simulation results of tethered chromophoric probes. We hope that our findings of interesting physical effects in the simple anthracene system will stimulate new experimental, simulation and theoretical investigations aimed at improved understanding of molecular diffusion. Our study of the relatively rigid anthracene molecule is also a good starting point on the way to simulating diffusion of more complex, flexible systems of biological interest, such as peptides.

We have used several different methods in our calculations of rotational diffusion coefficients, among which using angular velocity correlation functions in the molecular frame appears to be superior to the alternatives. This method yielded rotational reorientation times in best agreement with experimental results for the systems studied here. The angular velocity autocorrelation approach was also technically simplest, as it did not involve fitting of noisy data to assumed functional forms.

\section{ACKNOWLEDGMENTS}

This project was supported in part by a $\mathrm{K}^{*} \mathrm{STAR}$ NSF EPSCoR FIRST Award (KK), by the American Heart Association, Kansas Affiliate, Postdoctoral Fellowship No. KS95-F-7 (G.S.J.), and by NSF EPSCoR Grant No. OSR9255223 and a University of Kansas General Research Fund grant (C.K.J.). G.S.J. is an American Heart Association Fellow. K.K. and G.S.J. gratefully acknowledge the Kansas Institute for Theoretical and Computational Science and the University of Kansas Molecular Modeling and Graphics Laboratory for making available their computer resources for a part of this project. C.K.J. and S.W.P. thank Graham Fleming for sharing software for iterative least-squares fits of fluorescence decays. We would like to thank an anonymous referee for pointing out that the shape of anthracene is intermediate between an oblatelike and a prolatelike ellipsoid.

\footnotetext{
${ }^{1}$ H. J. Parkhurst, Jr. and J. Jonas, J. Chem. Phys. 63, 2698 (1975).

${ }^{2}$ J. Jonas and J. A. Akai, J. Chem. Phys. 66, 4956 (1977).

${ }^{3}$ G. T. Evans, J. Chem. Phys. 88, 5035 (1988).

${ }^{4}$ D. Ben-Amotz and J. M. Drake, J. Chem. Phys. 89, 1019 (1988).

${ }^{5}$ N. Tjandra, S. E. Feller, R. W. Pastor, and A. Bax, J. Am. Chem. Soc. 117, 12562 (1995).

${ }^{6}$ M. Terazima and N. J. Hirota, J. Chem. Phys. 98, 6257 (1993).

${ }^{7}$ G. G. Belford, R. L. Belford, and G. Weber, Proc. Natl. Acad. Sci. USA 69, 609 (1972).
} 
${ }^{8}$ J. R. Lakowicz, H. Cherek, B. P. Mailwal, and E. Gratton, Biochemistry 24, 376 (1985).

${ }^{9}$ C. Z. Wan, Q. Song, and C. K. Johnson, Chem. Phys. Lett. 210, 94 (1993).

${ }^{10}$ J. K. Moscicki, Y. K. Shin, and J. H. Freed, in EPR Imaging and In Vivo $E P R$, edited by R. R. Eaton et al. (1991).

${ }^{11}$ Y. K. Shin, U. Ewert, D. E. Budil, and J. H. Freed, Biophys. J. 59, 950 (1991).

${ }^{12}$ J. K. Moscicki, Y. K. Shin, and J. H. Freed, J. Chem. Phys. 99, 634 (1993).

${ }^{13}$ A. Einstein, Investigations on the Theory of Brownian Movement (Dover, New York, 1956).

${ }^{14}$ J. T. Hynes, R. Kapral, and M. Weinberg, J. Chem. Phys. 69, 2725 (1978).

${ }^{15}$ J. L. Dote and D. Kivelson, J. Phys. Chem. 87, 3889 (1983).

${ }^{16}$ D. Ben-Amotz and T. W. Scott, J. Chem. Phys. 87, 3739 (1987).

${ }^{17}$ W. Eimer and R. Pecora, J. Chem. Phys. 94, 2324 (1991).

${ }^{18}$ C. R. Cantor and P. R. Schimmel, Biophysical Chemistry: Techniques for the Study of Biological Structure and Function (Freeman, New York, 1980), Vol. II.

${ }^{19}$ F. Perrin, J. Phys. Radium 5, 497 (1934).

${ }^{20}$ F. Perrin, J. Phys. Radium 7, 1 (1936).

${ }^{21}$ C. M. Hu and R. Zwanzig, J. Chem. Phys. 60, 4345 (1974).

${ }^{22}$ J. L. Dote, D. Kivelson, and R. N. Schwartz, J. Phys. Chem. 85, 2169 (1981).

${ }^{23}$ G. K. Youngren and A. Acrivos, J. Chem. Phys. 63, 3846 (1975).

${ }^{24}$ R. J. Sension and R. M. Hochstrasser, J. Chem. Phys. 98, 2490 (1992).

${ }^{25}$ M. M. Tirado and J. G. De La Torre, J. Chem. Phys. 71, 2581 (1979).

${ }^{26}$ M. M. Tirado and J. G. De La Torre, J. Chem. Phys. 73, 1986 (1980).

${ }^{27}$ J. G. De La Torre and V. A. Bloomfield, Q. Rev. Biophys. 14, 81 (1981).

${ }^{28}$ M. M. Tirado, M. C. LopezMartinez, and J. G. De La Torre, J. Chem. Phys. 81, 2047 (1984).

${ }^{29}$ J. G. De La Torre, M. C. LopezMartinez, and M. M. Tirado, Biopolymers 23, 611 (1984)

${ }^{30}$ T. Tao, Biopolymers 8, 609 (1969).

${ }^{31}$ Y. R. Kim and R. M. Hochstrasser, J. Chem. Phys. 96, 9597 (1992).

${ }^{32}$ W. Brown and Z. Pu, Macromolecules 23, 5097 (1990).

${ }^{33}$ T. Dorfmuller, B. Daum, and A. Hanschmidt, J. Chem. Phys. 95, 813 (1991).

${ }^{34}$ J. Kanetakis and H. Sillescu, Chem. Phys. Lett. 252, 127 (1996).

${ }^{35}$ L. Vuillard, B. Roux, and A. Miller, Eur. J. Biochem. 191, 333 (1990).

${ }^{36}$ P. W. Kuchel and B. E. Chapman, J. Magn. Reson. 94, 574 (1991).

${ }^{37}$ U. Stark, F. Cebe, K. Meisegresch, and W. M. Warmuth, J. Mol. Liq. 52, 67 (1992).

${ }^{38}$ J. F. Ellena, L. S. Lepore, and D. S. Cafiso, FASEB J. 6, A505 (1992).

${ }^{39}$ H. Liu, F. Muller-Plathe, and W. F. van Gunsteren, J. Am. Chem. Soc. 117, 4363 (1995).

${ }^{40}$ Y. Zhang, R. M. Venable, and R. W. Pastor, J. Phys. Chem. 100, 2652 (1996).

${ }^{41}$ A. Brodka and T. W. Zerda, J. Chem. Phys. 104, 6313 (1996)

${ }^{42}$ P. E. Smith and W. F. van Gunsteren, J. Mol. Biol. 236, 629 (1994).

${ }^{43}$ Y. Hu and G. R. Fleming, J. Chem. Phys. 94, 3857 (1991).

${ }^{44}$ A. Luzar and D. Chandler, J. Chem. Phys. 98, 8160 (1993).

${ }^{45}$ D. Huppert, D. C. Douglass, and P. M. Rentzepis, J. Chem. Phys. 72, 2841 (1980).

${ }^{46}$ A. Bree and S. J. Katagiri, J. Mol. Spectrosc. 17, 24 (1965).

${ }^{47}$ A. Bree, S. J. Katagiri, and S. R. Suart, J. Chem. Phys. 44, 1788 (1966).

${ }^{48}$ G. J. Small, J. Chem. Phys. 52, 656 (1970).
${ }^{49}$ T. P. Carter and G. D. Gillespie, J. Phys. Chem. 86, 2591 (1982).

${ }^{50}$ W. R. Lambert, P. M. Felker, J. A. Syage, and A. H. Zewail, J. Chem. Phys. 81, 2195 (1984).

${ }^{51}$ G. N. R. Triphati and M. R. Fisher, Chem. Phys. Lett. 104, 297 (1984).

${ }^{52}$ A. Bree, A. Leyderman, and C. Taliani, J. Mol. Struct. 142, 151 (1986).

${ }^{53}$ J. Wolf and G. Hohlneicher, Chem. Phys. 181, 185 (1994).

${ }^{54}$ G. S. Jas, C. Wan, K. Kuczera, and C. K. Johnson, J. Phys. Chem. 100, 11857 (1996)

${ }^{55}$ G. S. Jas and K. Kuczera, Chem. Phys. 214, 229 (1997).

${ }^{56}$ M. Lettenberger, F. Emmerling, N. H. Gottfried, and A. Laubereau, Chem. Phys. Lett. 95, 813 (1995).

${ }^{57}$ A. M. Williams, Y. Jiang, and D. Ben-Amotz, Chem. Phys. 180, 119 (1994).

${ }^{58}$ A. J. Cross and G. R. Fleming, Biophys. J. 46, 45 (1984)

${ }^{59}$ B. R. Brooks, R. Bruccoleri, B. Olafson, D. States, S. Swaminathan, and M. Karplus, J. Comput. Chem. 4, 187 (1983).

${ }^{60}$ A. D. Mackerell, Jr., M. Field, S. Fischer, and M. Karplus (unpublished).

${ }^{61}$ M. J. Frisch, G. W. Trucks, M. Head-Gordon, P. M. W. Gill, W. Wong, J. B. Foresman, B. G. Hohnson, H. B. Schlegel, M. A. Robb, E. S. Replogle, R. Gomperts, J. L. Andres, K. Raghavachari, J. S. Binkley, C. Gonzales, R. L. Martin, D. S. Fox, D. J. Defrees, J. Barker, C. Gonzales, R. L. Martin, D. S. Fox, D. J. Defrees, J. Barker, J. J. P. Stewart, and J. A. Pople, in Gaussian 92, Revision C (Gaussian Inc., Pittsburgh, PA, 1992).

${ }^{62}$ S. R. Cox and D. E. Williams, J. Comput. Chem. 2, 204 (1981).

${ }^{63}$ G. S. Jas and K. Kuczera (unpublished).

${ }^{64}$ CRC Handbook of Chemistry and Physics, 66th ed., edited by R. C. Weast (CRC Press, Cleveland, OH, 1985-1986).

${ }^{65}$ R. R. Dreisbach, Anal. Ed. 160 (1940).

${ }^{66}$ J. P. Ryckaert, G. Ciccotti, and H. J. C. Berendsen, J. Comput. Phys. 23, 327 (1977).

${ }^{67}$ E. Barth, K. Kuczera, B. Leimkuhler, and R. D. Skeel, J. Comput. Chem. 16, 1192 (1995).

${ }^{68}$ L. Verlet, Phys. Rev. 159, 98 (1967).

${ }^{69}$ J. McConnell, Rotational Brownian Motion and Dielectric Theory (Academic, London, 1980).

${ }^{70}$ R. Zwanzig and M. Bixon, Phys. Rev. A 2, 2005 (1970).

${ }^{71}$ L. P. Hwang and J. H. Freed, J. Chem. Phys. 63, 118 (1975).

${ }^{72}$ E. Ehrenberg and R. Rigler, Chem. Phys. Lett. 14, 539 (1972).

${ }^{73}$ T. J. Chaung and K. B. Eisenthal, J. Chem. Phys. 57, 5094 (1972).

${ }^{74}$ D. A. McQuarrie, Statistical Mechanics (Harper \& Row, New York, 1976).

${ }^{75}$ T. Chalikian and K. Breslauer, Biopolymers 39, 619 (1996).

${ }^{76}$ J. A. Riddick, W. B. Bunger, and T. K. Sakand, Organic Solvents: Physical Properties and Methods of Purification (Wiley, New York, 1986).

${ }^{77}$ E. L. Quitevis and M. L. Horng, J. Phys. Chem. 94, 5684 (1990).

${ }^{78}$ R. D. Bessire and E. L. Quitevis, J. Phys. Chem. 98, 13083 (1994).

${ }^{79}$ D. R. Bauer, G. R. Alms, J. I. Brauman, and R. Pecora, J. Chem. Phys. 59, 5321 (1973)

${ }^{80}$ D. R. Bauer, J. I. Brauman, and R. Pecora, J. Am. Chem. Soc. 96, 6840 (1974).

${ }^{81}$ L. X. Q. Chen, R. A. Engh, and G. R. Fleming, J. Phys. Chem. 92, 1066 (1988).

${ }^{82}$ QUANTA by Molecular Simulations, Inc., San Diego, CA.

${ }^{83}$ P. M. Andrews, B. A. Pryor, P. M. Palmer, and M. R. Topp, Chem. Phys. Lett. 265, 224 (1997). 